\title{
Decentralised Model Predictive Control of Electric Vehicles Charging
}

\author{
Alessandro Di Giorgio Member, IEEE, Alessandro Giuseppi* Student Member, IEEE, Roberto Germanà and \\ Francesco Liberati Member, IEEE
}

\begin{abstract}
This paper presents a decentralised control strategy for the management of simultaneous charging sessions of electric vehicles. The proposed approach is based on the model predictive control methodology and the Lagrangian decomposition of the constrained optimization problem which is solved at each sampling time. This strategy allows the computation of the charging profiles in a decentralised way, with limited information exchange between the electric vehicles. The simulation results show the potential of the proposed approach in relation to the problem of shaving the aggregated power withdrawal from the electricity distribution grid, while still satisfying drivers' preferences for charging.
\end{abstract}

\section{INTRODUCTION}

In recent years the electric mobility has grown in popularity, pushed by the need to overcome the problems of pollution, depletion of natural oil and fossil fuel reserves, and rising petrol costs. The automotive industry is also motivated to adopt cleaner and more sustainable technologies by the governmental regulations and international agreements (see e.g. [1][2]). As largely recognized, massive Electric Vehicles (EVs) charging represents a concern for the operation of electricity distribution grids, but also an opportunity, due to the possibility of exploiting the flexibility offered by the vehicles during charging. Over the last decade, this remark has motivated the investigation of several methodologies for the control of the EVs charging process according to a pletora of grid and drivers' requirements. Among the criteria used for classifying the control approaches, the centralized/decentralized nature of the control plays an important role, since it reflects two different visions of the new electromobility paradigm. On the one hand the electric companies, as responsible for the secure operation of the electric network or interested players in the electricity markets, are interested in managing the charging processes in a centralized way; on the other car manufacturers are also interested in using the EVs as a mean for enabling new business models and avoiding to share proprietary information related to the EVs charger with other market players, which brings to the idea of a decentralized control. Both these two classes of control have been proposed in the relevant literature for different purposes. Centralized control strategies have been developed for minimizing the peak load and avoiding distribution network issues [3], [4], reducing the power losses [5], avoiding network congestion

* coressponding author

A. Di Giorgio, A. Giuseppi, R. Germanà and F. Liberati are with the Department of Computer, Control and Management Engineering Antonio Ruberti, at Sapienza University of Rome, Via Ariosto 25, 00185, Rome, Italy, e-mail: \{digiorgio,giuseppi,germana,liberati\}@diag.uniroma1.it.

This work is partially supported by the H2020 project 5G-SOLUTIONS, Grant agreement ID: 856691. and lower $\mathrm{CO} 2$ emissions [6], minimizing the total cost of energy for users [7], maximizing aggregator profits and guarantee voltage regulation [8], tracking of target load curves [9][10], deliver balancing [11][12] and frequency regulation services [13]. Several studies also explore the possibility of utilising the EV batteries as a distributed Energy Storage Systems [14] to offer additional seriveces to the grid, as service restoration [15] and resiliency improvements [16].

Similarly, decentralized control strategies have been proposed for avoiding overloads [17] [18], minimizing the total cost of energy for users and reducing power losses [19], maximizing aggregator profits [20][21], frequency regulation and integration of renewable energy sources [22], for reference power tracking [23], etc. An in-depth analysis of the many pursued objectives and proposed algorithms can be found for example in the comprehensive reviews [24] and [25].

This paper targets a reference scenario consisting of a load area, namely a node of the distribution grid, equipped with a set of charging stations for the delivery of charging services. The paper proposes a decentralised control strategy aimed at maximizing the margin between the utility deriving to the drivers from the delivery of the charging service, and the cost for the operator deriving from the aggregated charging power withdrawal. The proposed control is based on a Model Predictive Control (MPC) framework, which embeds a constrained optimization problem, taking into account both grid and drivers' requirements, which can be decomposed through Lagrangian relaxation. Several centralised control solutions based on MPC can be found for similar case studies in the literature [26]-[28], while in this work the computation of the charging load curves is performed by agents working at the level of each single EV. Furthermore, the information exchanged with grid players is restricted to the computed load curve and energy price feedback from the market, elaborated according to the charging infrastructure congestion. Comparable scenarios have also been addressed by decentralised solutions, as in [29], in which a Mixedinteger optimisation formulation is utilised to model the optimization problem in which the various users trade their energy flexibility, in [30], where an aggregator agent dynamically modifies the energy pricing signal to steer a set of EVs behaviour, and in [31] where a game-theoretic strategy is employed. In the envisaged scenario, the main innovation of this work are:

- The predictive and dynamic nature of the charging session scheduling, as the MPC scheme allows to attain optimal performances over its prediction window and, thanks to its receding horizon paradigm, it allows the 
system to easily respond to new charging requests.

- The focus on decentralisation, as the amount of information exchanged between the agents is limited thanks to the Lagrangian Dual Decomposition approach.

- The focus on the trade-off between economic performances and user satisfaction, captured by the utility and cost functions considered for the optimisation.

The remainder of the paper is organized as follows. Section II recalls the fundamental concept of the MPC methodology and describes at high level how it is applied in this paper. Section III presents the formalization of the open loop optimal control problem at the basis of the MPC scheme. Section IV describes the decentralized solving procedure of the problem. Section V presents and discusses the simulation results and, finally, section VI reports the conclusions and directions for future works.

\section{Control methodology}

The proposed controller is designed based on the discretetime MPC methodology [32]. Discrete-time MPC is an optimization-based technique in which, at the generic discrete time $t$, the plant control signals are computed by solving a constrained optimization problem, usually referred to as open loop control problem, defined in a time window $N$ steps in the future (i.e. $[t, t+N-1]$ ); the first sample of the computed control signals is applied to the plant (the remaining sequence is discarded) and then the process is reiterated at time $t+1$. The generic optimization problem at time $t$ is built based on the feedback of the state plant at $t$, so that the closed loop properties of MPC arise from the combination of state feedback and continuous reoptimization.

MPC is one of the advanced control techniques most used in the industrial control practice, mainly because of its native ability and easiness in managing multi-variable constrained control. Also, great design flexibility is offered by the possibility of selecting and tuning the objective function and the constraints to be included in the optimization problem.

In standard MPC, the objective function terms are selected with the aim of stabilizing the plant state around a desired reference state, while minimizing the control effort. A distinctive aspect of the MPC here proposed is that the objective is designed to optimize the user satisfaction and the economical operation of the system.

In this paper the control signals computed by the controller at each time $t$ are the charging power of the EVs; though the power flowing at the point of connection is a problem variable, in principle it does not represent a control variable, since it results from the EVs controls in the load area. The feedback signals retrieved at each $t$ for computing such an optimal control are the current State of Charge (SOC) of the EVs in the network. Further key input to the MPC controller at $t$ are the boundary conditions and preferences characterizing the requests for the charging service, more specifically i) the arrival time at the charging station, ii) the initial SOC, iii) the departure time and iv) the desired state of charge; also the EVs and the point of connection of the load area with the grid are characterized by their own technical and economic data.

Despite the potential large amount of critical data being part of the open loop optimal control problem, a distinctive aspect of this work is the one of being solved in a decentralised way; the information exchanged by the EVs and the operator is restricted to power and energy price, while technical and economic information related to the equipment are not shared, then preserving user privacy and sensitive car manufacturers' data.

\section{CONTROL PROBLEM FORMALIZATION}

\section{A. Preliminaries}

This section presents the open loop optimal control problem at the basis of the proposed MPC scheme. In what follows $t$ will denote the current time, $T_{t}=\{t, t+1, \cdots, t+N-1\}$ the set of discrete time instants within the control window starting from $t, \tau$ the generic time instant within the set. The set $R_{t}$ is introduced to denote the EVs connected to a charging station at $t$ and consequently being part of the control problem.

\section{B. Agents behavioural models}

The behaviour of the agents being part of the control problem is modeled taking advantage of the concept of utility and cost functions usually applied in the context of microeconomics and resource management in telecomunication networks [33], [34].

Each EV $r \in R_{t}$ is modeled by a utility function $U_{r}\left(p_{r}(\tau / t)\right)$ which represents the level of satisfaction for the withdrawal of the charging power $p_{r}(\tau / t)$ at time $\tau \in T_{t}$, The utility function is requested to satisfy three properties: (i) it has to be continuous, (ii) monotonically increasing and (iii) strictly concave. From the modeling perspective, these requirements reflect the fact that the level of satisfaction of each driver grows up continuously with the level of charging power and is subject to saturation; from the theoretical point of view, this natural choice brings to the definition of a convex optimization problem. In particular, the utility function of the $\mathrm{r}$-th $\mathrm{EV}$ is here chosen as

$$
U\left(p_{r}(\tau / t)\right)=w_{r} \log \left(1+p_{r}(\tau / t)\right) \quad \forall \tau \in T_{t}
$$

where $w_{r}$ is a weight introduced to differentiate the behaviour of the drivers.

The system operator is modeled in relation to the effect that multiple charging sessions have in terms of aggregated power withdrawal at the point of connection between the load area and the distribution grid. A cost function $C(P(\tau / t))$ is then introduced, where $P(\tau / t)$ is the power flowing at the point of connection at time $\tau \in T_{t}$. This cost function is requested to meet the following requirements: $(i)$ it has to be continuous, (ii) monotonically increasing and (iii) strictly convex; as before, these are natural requirements, introduced to penalize the peaks of power withdrawal from the grid. Specifically, the cost function is here chosen as

$$
\begin{aligned}
C(P(\tau / t)) & =\alpha(P(\tau / t))^{2}+ \\
& +\delta(P(\tau / t)-P(\tau-1 / t))^{2} \quad \forall \tau \in T_{t}
\end{aligned}
$$


where $\alpha$ and $\delta$ are proper weights. The first term represents a penalty for excessive power withdrawal from the grid, while the second one is a ramping rate term aimed at avoiding fast variation of the power at the point of connection.

The utility and cost functions model counteracting requirements from different players. On the one hand, the drivers are interested in disposing of the highest possible level of charging power, on the other hand, the owner of the infrastructure is interested in minimizing the deriving operational cost. Then a trading mechanism is needed for establishing a proper trade-off allowing to meet driver needs for charging while guaranteeing acceptable operating conditions for the electrical infrastructure.

\section{The open loop optimal control problem}

In order to establish the required trade-off, the social welfare, defined as the difference between the total utility and cost in the area, is evaluated over all the control horizon, and the optimization criterion is consequently formalized as

$$
\max _{\substack{p_{r}(\tau / t), P(\tau / t) \\ \forall \tau \in T_{t}}} \sum_{\tau \in T_{t}}\left\{\sum_{r \in R_{t}} U_{r}\left(p_{r}(\tau / t)\right)-C(P(\tau / t))\right\}
$$

The optimal control sequences $p_{r}^{*}$ and $P^{*}$ are subject to three classes of constraints, taking into account the overall power balance, technical limitations and preferences at the level of the single EVs and limitations of load area equipment. As far as it concerns the overall behaviour of the agent at load area level, the power balance has to be guaranteed at each $\tau \in T_{t}$, which is modeled as

$$
\sum_{r \in R_{t}} p_{r}(\tau / t)=P(\tau / t), \forall \tau \in T_{t}
$$

At EV level the feasibility of the charging session has to be guaranteed both in terms of allowed power withdrawal and driver preferences satisfaction. According to the IEC 61851 international standard, only the values of current exceeding the threshold of $6 \mathrm{~A}$ are allowed for charging; also the maximum current is upper bounded depending on the EV and the charging station technology, so that the power is limited accordingly as

$$
p_{r}^{\text {min }}<p_{r}(\tau / t)<p_{r}^{\text {max }}, \forall r \in R_{t}, \forall \tau \in T_{t}
$$

The driver preferences for charging are taken into account as follows. Let $e_{r}(\tau / t)=x_{r}(\tau / t)-x_{r}^{\text {des }}$ denote the deviation of the state of charge $x_{r}(\tau / t)$ of EV $r \in R_{t}$ at time $\tau \in T_{t}$ from the desired state of charge $x_{r}^{\text {des}}$; this error signal is subject to the dynamics

$e_{r}(\tau+1 / t)=e_{r}(\tau / t)-\left(1-\xi_{r}\right) \Delta t p_{r}(\tau / t), \forall \tau \in T_{t}, \forall r \in R_{t}$ $e_{r}(t / t)=e(t), \forall r \in R_{t}$

where $\Delta t$ denotes the sampling time, $\xi_{r}$ the conversion losses coefficient and $e_{r}(t)=x_{r}(t)-x_{r}^{\text {des }}$ the actual error at current time $\mathrm{t}$, evaluated using the feedback signal $x_{r}(t)$ and the desired state of charge $x_{r}^{\text {des }}$. The latter, which is in principle different from the maximum capacity of the battery $x_{r}^{\max }$, has to be guaranteed at the departure time $t_{r}^{\text {dep }}$ chosen by the driver, so that

$$
e_{r}\left(t_{r}^{d e p} / t\right)=0, \forall r \in R_{t}
$$

As expressed in (4), the aggregated charging power is provided by the distribution grid at the point of connection (typically through a dedicated medium voltage to low voltage substation); due to the limited power rating of the transformers the power $P$ is bounded as follows:

$$
P^{\min } \leq P(\tau / t) \leq P^{\max }, \forall \tau \in T_{t}
$$

In the light of the above, the open loop optimal control to be solved at each iteration of the MPC scheme can be stated as follows.

Open loop EVs charging control problem. For a given load area characterized by operational cost (2), hosting a set $R_{t}$ of charging EVs having utility (1) and preferences $\left(x^{\text {des }}, t^{\text {dep }}\right)$, solve (3), subject to the dynamics (6), control constraints (4), (5), (8) and state constraints (7).

Remark 1 In order for the optimisation problem to be feasible, it is required that all session requests have to be checked for consistency in terms of their departure time and desired state of charge, in such a way that they are compatible with the maximum EV charging power. Furthermore, the aggregated charging session shall not require, over their time windows, more energy than the maximum output of the load area.

\section{DECENTRALIZED SOLVING PROCEDURE}

The problem previously formalized is solved in a decentralised way taking advantage of the Lagrangian theory (in particular the duality theory [35]) and the specific form of the target function and constraints. For convenience of notation, as customary in the MPC literature, a vector is introduced to denote in a compact form each variable which is defined over the whole control horizon; in particular $\boldsymbol{p}_{\boldsymbol{r}}(t)=\operatorname{col}\left(p_{r}(t / t), p_{r}(t+1 / t), \ldots, p_{r}(t+N-1 / t)\right)$ and $\boldsymbol{P}(t)=\operatorname{col}(P(t / t), P(t+1 / t), \ldots, P(t+N-1 / t))$ will denote the r-th EV and the load area power over the period respectively.

The Lagrangian function is introduced by combining the target function (3) and the power balance constraint (4) - the only one explicitly matching EVs and load area variables as

$$
\begin{aligned}
L\left(\boldsymbol{p}_{\boldsymbol{r}}(t), \boldsymbol{P}(t), \boldsymbol{\lambda}(t)\right)= \\
=\sum_{\tau \in T_{t}}\left\{\sum_{r \in R_{t}} U_{r}\left(p_{r}(\tau / t)\right)-C(P(\tau / t))\right\}+ \\
\quad-\sum_{\tau \in T_{t}} \lambda(\tau / t)\left\{\sum_{r \in R_{t}} p_{r}(\tau / t)-P(\tau / t)\right\}
\end{aligned}
$$

where $\lambda(t)=\operatorname{col}(\lambda(t / t), \lambda(t+1 / t), \ldots, \lambda(t+N-1 / t))$ is the vector of Lagrangian multipliers; as it will be clarified in the following, the multipliers represent an indicator of the energy price in the load area. 
The dual problem is defined as

$$
\min _{\boldsymbol{\lambda}(t)>0} D(\boldsymbol{\lambda}(t))
$$

where

$$
D(\boldsymbol{\lambda})=\max _{\boldsymbol{p}_{\boldsymbol{r}}(t), \boldsymbol{P}(t)} L\left(\boldsymbol{p}_{\boldsymbol{r}}(t), \boldsymbol{P}(t), \boldsymbol{\lambda}(t)\right),
$$

subject to constraints (5) - (8).

The Lagrangian function can be decomposed as

$$
L\left(\boldsymbol{p}_{\boldsymbol{r}}(t), \boldsymbol{P}(t), \boldsymbol{\lambda}(t)\right)=\sum_{r \in R_{t}} f\left(\boldsymbol{p}_{\boldsymbol{r}}(t), \boldsymbol{\lambda}(t)\right)+g(\boldsymbol{P}(t), \boldsymbol{\lambda}(t))
$$

where

$$
f\left(\boldsymbol{p}_{\boldsymbol{r}}(t), \boldsymbol{\lambda}(t)\right)=\sum_{\tau \in T_{t}}\left\{U_{r}\left(p_{r}(\tau / t)\right)-\lambda(\tau / t) p_{r}(\tau / t)\right\}
$$

and

$$
g(\boldsymbol{P}(t), \boldsymbol{\lambda}(t))=\sum_{\tau \in T_{t}}\{\lambda(\tau / t) P(\tau / t)-C(P(\tau / t))\}
$$

Taking advantage of this property the dual problem can be decomposed in subproblems, logically related to different agents, and solved iteratively. The generic $k$-th iteration of the procedure is as follows.

In the first step each EV and the operator compute the respective optimal power, for a given value of multipliers $\lambda_{k}(t)$. Each EV solves the subproblem

$$
\max _{\boldsymbol{p}_{\boldsymbol{r}}(t)} f\left(\boldsymbol{p}_{\boldsymbol{r}}(t), \boldsymbol{\lambda}_{k}(t)\right)
$$

subject to constraints (5) - (7). This means finding, for a given $\lambda_{k}(t)$, the optimal charging power $\boldsymbol{p}_{\boldsymbol{r}_{k}}(t)$ over the time which maximizes the margin among utility and cost, while respecting technical constraints and charging preferences. Notice that, due to the assumption made on the utility function (1), the EV subproblem is a convex optimization problem.

Similarly the operator solves the subproblem

$$
\max _{\boldsymbol{P}(t)} g\left(\boldsymbol{P}(t), \boldsymbol{\lambda}_{k}(t)\right)
$$

subject to constraint (8). Again, this means finding, for a given $\lambda_{k}(t)$, the optimal power curve $\boldsymbol{P}_{k}(t)$ at the interface with the distribution grid which maximizes the margin among the benefit and cost, while keeping feasible operation of operator's equipment. As before, due to the properties of the utility function (2), the operator subproblem is convex.

In the second step of the procedure, once the powers $\boldsymbol{p}_{\boldsymbol{r}_{k}}(t)$ and $\boldsymbol{P}_{k}(t)$ are known, the Lagrangian multipliers are updated following the anti-gradient of $D(\boldsymbol{\lambda})$. The updating rule is then

$$
\boldsymbol{\lambda}_{k+1}(t)=\max \left(\boldsymbol{\lambda}_{k}(t)-\gamma \nabla \boldsymbol{D}_{k}, \mathbf{0}\right)
$$

where

$$
\nabla \boldsymbol{D}=\boldsymbol{P}_{k}(t)-\sum_{r \in R_{t}} \boldsymbol{p}_{\boldsymbol{r}_{k}}(t)
$$

and the step $\gamma$ is chosen according to the Armijo's rule. Notice that $\boldsymbol{\lambda}_{k}(t)$ evolves according to the imbalance between the aggregated charging load and the power supply in the load
TABLE I

SimUlation PARAMETERS

\begin{tabular}{cc}
\hline Parameter & Value \\
\hline$\Delta t$ & $5 \min$ \\
$N$ & 36 \\
$\alpha, \delta$ & $0.5,0.1$ \\
$\epsilon$ & 0.1 \\
$U(p)$ & $\omega \log (1+p)$ \\
$\omega$ & 15 \\
$\xi$ & 0.05 \\
$p^{\text {max }}, p^{\text {min }}$ & $22 k W, 0 k W$ \\
$x^{\text {max }}$ & $24 k W h$ \\
$P^{\text {max }}, P^{\text {min }}$ & $200 k W, 0 k W$ \\
\hline
\end{tabular}

TABLE II

Simulation 1 - Charging Sessions

\begin{tabular}{cccc}
\hline EV ID & Start time & End Time & Initial SOC [\%] \\
\hline 1 & $00: 00$ & $04: 00$ & 12.5 \\
2 & $00: 00$ & $05: 00$ & 25 \\
3 & $00: 00$ & $03: 30$ & 29 \\
4 & $03: 00$ & $07: 00$ & 21 \\
\hline
\end{tabular}

area; specifically the values of the Lagrangian multipliers increase as the demand outbalance the supply and vice-versa, so that the multipliers can be interpreted as an indicator of the energy price in the load area.

The two-steps procedure is repeated using $\lambda_{k+1}(t)$, until a $k^{*}$ exists for which the exit condition

$$
\left\|\nabla D_{k^{*}}\right\|<\epsilon
$$

is satisfied, for an arbitrary small positive real number $\epsilon$. If (19) holds, the balance (4) between demand and supply is reached in practice, and the optimal solution of the dual problem is achieved. The point $\left(\boldsymbol{p}_{\boldsymbol{r}}{ }^{*}(t), \boldsymbol{P}^{*}(t), \boldsymbol{\lambda}^{*}(t)\right)=$ $\left(\boldsymbol{p}_{\boldsymbol{r}_{k^{*}}}(t), \boldsymbol{P}_{k^{*}}^{\boldsymbol{l}}(t), \boldsymbol{P}_{k^{*}}^{\boldsymbol{s}}(t), \boldsymbol{\lambda}_{k^{*}}(t)\right)$ represents the optimal control and price sequences over the control horizon, according to the boundary conditions characterizing the load area at current time $t$; consequently $\left(p_{r}(t), P(t), \boldsymbol{\lambda}(t)\right)=$ $\left.\left(p_{r}^{*}(t / t), P^{*}(t / t)\right), \lambda^{*}(t / t)\right)$ is the control and the price actually applied to the plant at current time $t$.

\section{Simulation Results and Discussion}

The proposed control algorithm has been validated at simulation level in order to provide a preliminary proof of concept. The simulation framework has been built in Matlab, leveraging Matlab built-in solver for the solution of agents' optimization problems; the simulation parameters are specified in Table I, while the simulation scenarios are reported in Table II and III. For simplicity, the desired final SOC has been set to $90 \%$ for all the charging sessions, due to the fact that this value represents the bound beyond which the validity of model (6) becomes questionable.

The first simulation is intended to show how the algorithm works in a simplified scenario where it is straightforward to check for its effectiveness. Fig. 1 shows the aggregated charging power in the (a) uncontrolled case, in which the charging service is provided at rated power starting at the 
TABLE III
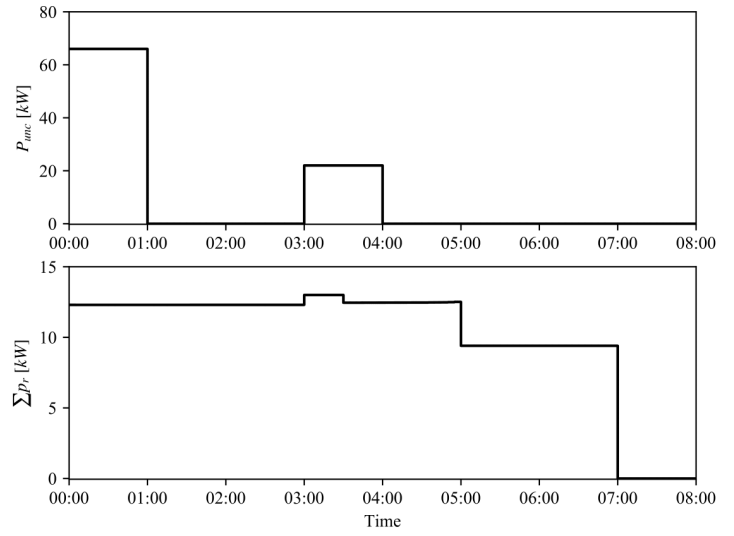

Fig. 1. Simulation 1 - Aggregated charging power in the (a) uncontrolled and (b) controlled case.

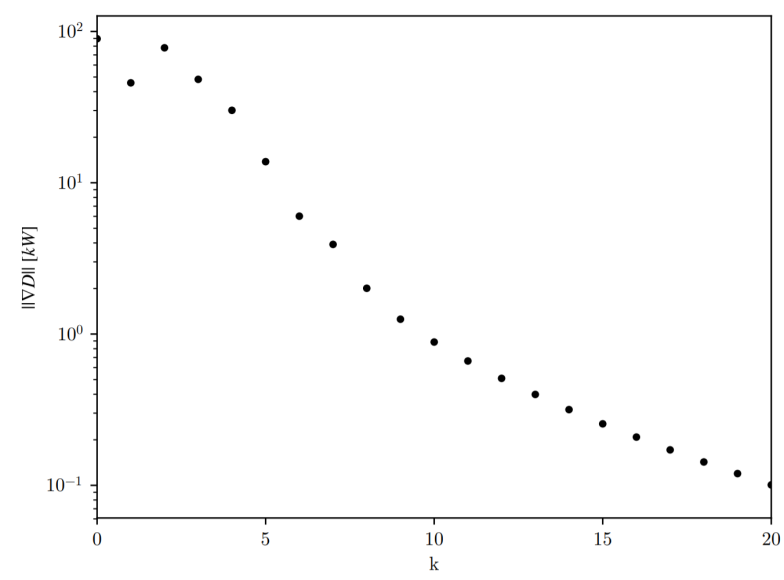

Fig. 2. Simulation 1 - Evolution of $\|\nabla D\|$ for the optimization performed at $2: 55$.

time of arrival, and in the (b) controlled one. It is immediate to see how the control distributes the charging power over the time in order to smooth the aggregated power profile. As far as concerns the decentralised optimization occurring at each iteration of the MPC scheme, Fig. 2 reports an example of the evolution of $\|\nabla D\|$ over the proposed decentralised optimization procedure: it is seen that $\|\nabla D\|$ has a fairly regular behaviour, and the convergence is achieved after 20 iterations of the procedure.

Simulation 2 is characterized by a more complex and realistic scenario, in which the proposed control better show its potential. In absence of control, the charging requests produce a highly variable aggregated charging power characterized by peaks reaching approximately $90 \mathrm{~kW}$ (Fig. 3 ); the proposed control allows to significantly mitigate the variability of the aggregated power withdrawal while allowing to steer the SOC errors of all the charging sessions to zero (Fig. 4). Finally Fig. 5 reports the evolution of $\|\nabla D\|$ for the optimization procedure occurring at 3:45, a congested time in which $9 \mathrm{EVs}$ charge simultaneously. The convergence is
Simulation 2 - ChaRgING SESSIONS

\begin{tabular}{cccc}
\hline EV ID & Start time & End Time & Initial SOC [\%] \\
\hline 1 & $00: 15$ & $05: 15$ & 10 \\
2 & $00: 30$ & $04: 30$ & 10 \\
3 & $00: 45$ & $05: 00$ & 16 \\
4 & $01: 00$ & $02: 15$ & 10 \\
5 & $01: 30$ & $06: 00$ & 62 \\
6 & $02: 00$ & $07: 00$ & 41 \\
7 & $02: 45$ & $06: 45$ & 16 \\
8 & $03: 00$ & $08: 00$ & 10 \\
9 & $03: 00$ & $05: 00$ & 16 \\
10 & $03: 15$ & $04: 45$ & 10 \\
11 & $04: 00$ & $09: 00$ & 16 \\
12 & $05: 45$ & $10: 45$ & 13 \\
13 & $06: 00$ & $11: 00$ & 13 \\
14 & $07: 00$ & $10: 30$ & 13 \\
15 & $07: 00$ & $11: 00$ & 13 \\
16 & $07: 00$ & $11: 30$ & 16 \\
17 & $08: 30$ & $11: 00$ & 16 \\
18 & $08: 45$ & $11: 45$ & 16 \\
19 & $09: 00$ & $11: 45$ & 16 \\
20 & $09: 30$ & $11: 45$ & 16 \\
\hline
\end{tabular}
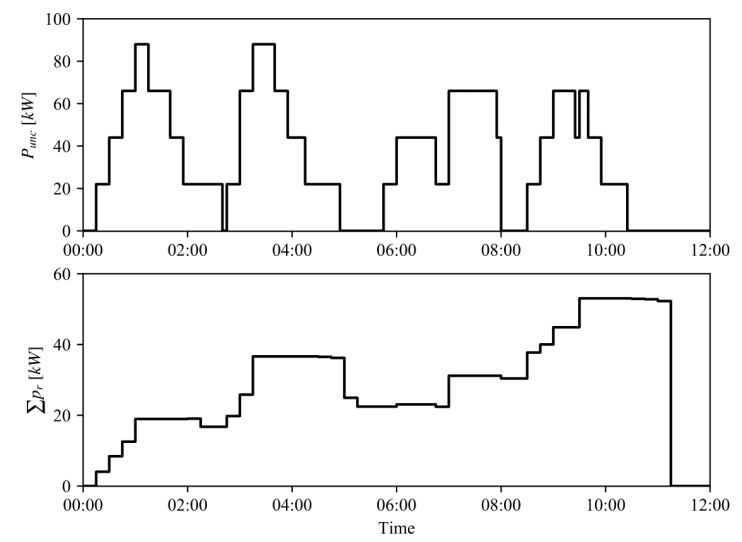

Fig. 3. Simulation 2 - Aggregated charging power in the (a) uncontrolled and (b) controlled case.

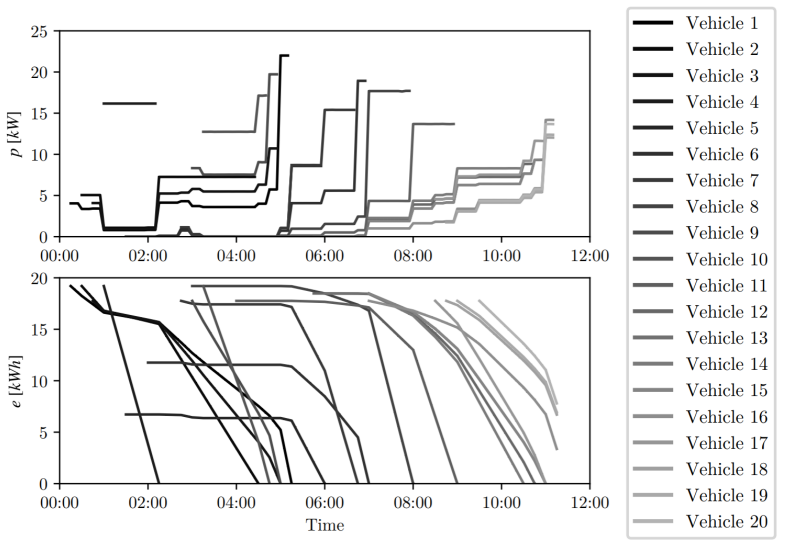

Fig. 4. Simulation 2 - Evolution of (a) power and (b) SOC for each charging session. 


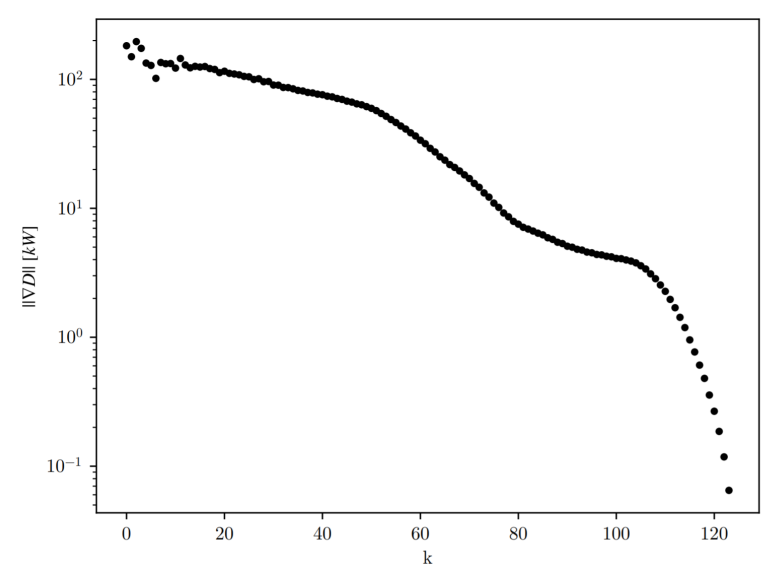

Fig. 5. Simulation 2 - Evolution of $\|\nabla D\|$ for the optimization performed at $3: 45$.

achieved in approximately 120 iterations; though this value may appear quite high for an implementation of the method in a real system, it is important to remark that it is affected by the choice of $\epsilon$ and the sampling time; moreover, due to the low complexity of the optimization problem solved by each agent (with solving times in the range of a second or less), it can be concluded that the proposed method is compatible with a real time application characterized by the proposed sampling time.

\section{CONCLUSIONS}

In this paper a real time decentralised control strategy for electric vehicles charging has been presented. The decentralised control mechanism is based on model predictive control methodology and Lagrangian decomposition of the optimization problem at its basis. The simulation results show the potential of the proposed approach, which can be implemented in practice in scenarios where the sampling time of the control action is in the typical range of power systems metering and scheduling applications. Possible directions for this work consider the integration of energy storage systems in the decentralised framework, the improvement of convergence performance and its theoretical validation.

\section{ACKNOWLEDGMENT}

The authors gratefully acknowledge Dr. R. Coccioli, Dr. G. Di Segni and Eng. A. Di Maria for the fruitful discussions over the paper's matters and the support in setting up the simulation environment. A special Acknowledgment goes to the CRAT team involved in the project $\mathrm{H} 2020$ 5G-SOLUTIONS.

\section{REFERENCES}

[1] European Parliament, Council of the European Union, "Directive (EU) 2018/844 of the European Parliament and of the Council of 30 May 2018 amending Directive 2010/31/EU on the energy performance of buildings and Directive 2012/27/EU on energy efficiency (Text with EEA relevance)," Official Journal of the European Union, vol. L 156, p. 75-91, 19.6.2018. [Online]. Available: http://data.europa.eu/eli/dir/2018/844/oj
[2] — "Directive 2009/33/EC of the European Parliament and of the Council of 23 April 2009 on the promotion of clean and energy-efficient road transport vehicles (Text with EEA relevance)," Official Journal of the European Union, vol. L 120, p. 5-12, 15.5.2009. [Online]. Available: https://eur-lex.europa.eu/legal-content/EN/ALL/ ?uri=CELEX:32009L0033

[3] J. Kang, S. J. Duncan, and D. N. Mavris, "Real-time scheduling techniques for electric vehicle charging in support of frequency regulation," Procedia Computer Science, vol. 16, pp. 767 - 775, 2013, 2013 Conference on Systems Engineering Research. [Online]. Available: http://www.sciencedirect.com/science/article/pii/S1877050913000811

[4] A. Di Giorgio, F. Liberati, R. Germana, M. Presciuttini, L. R. Celsi, and F. Delli Priscoli, "On the control of energy storage systems for electric vehicles fast charging in service areas," in 2016 24th Mediterranean Conference on Control and Automation (MED), June 2016, pp. 955960.

[5] S. Deilami, A. S. Masoum, P. S. Moses, and M. A. S. Masoum, "Realtime coordination of plug-in electric vehicle charging in smart grids to minimize power losses and improve voltage profile," IEEE Transactions on Smart Grid, vol. 2, no. 3, pp. 456-467, Sep. 2011.

[6] P. Richardson, D. Flynn, and A. Keane, "Local versus centralized charging strategies for electric vehicles in low voltage distribution systems," IEEE Transactions on Smart Grid, vol. 3, no. 2, pp. 10201028, June 2012.

[7] D. T. Nguyen and L. B. Le, "Joint optimization of electric vehicle and home energy scheduling considering user comfort preference," IEEE Transactions on Smart Grid, vol. 5, no. 1, pp. 188-199, Jan 2014.

[8] E. Sortomme and M. A. El-Sharkawi, "Optimal charging strategies for unidirectional vehicle-to-grid," IEEE Transactions on Smart Grid, vol. 2, no. 1, pp. 131-138, March 2011.

[9] A. D. Giorgio, F. Liberati, and S. Canale, "Electric vehicles charging control in a smart grid: A model predictive control approach," Control Engineering Practice, vol. 22, pp. 147 - 162, 2014.

[10] M. C. Kisacikoglu, F. Erden, and N. Erdogan, "Distributed control of pev charging based on energy demand forecast," IEEE Transactions on Industrial Informatics, vol. 14, no. 1, pp. 332-341, Jan 2018.

[11] F. Liberati, A. Mercurio, L. Zuccaro, A. Tortorelli, and A. Di Giorgio, "Electric vehicles charging load reprofiling," 2014, pp. 728-733.

[12] C. L. Floch, E. C. Kara, and S. Moura, "Pde modeling and control of electric vehicle fleets for ancillary services: A discrete charging case," IEEE Transactions on Smart Grid, vol. 9, no. 2, pp. 573-581, March 2018.

[13] F. Soares, P. R. Almeida, and J. P. Lopes, "Quasi-real-time management of electric vehicles charging," Electric Power Systems Research, vol. 108, pp. 293 - 303, 2014. [Online]. Available: http://www.sciencedirect.com/science/article/pii/S0378779613003210

[14] F. Mwasilu, J. J. Justo, E.-K. Kim, T. D. Do, and J.-W. Jung, "Electric vehicles and smart grid interaction: A review on vehicle to grid and renewable energy sources integration," Renewable and Sustainable Energy Reviews, vol. 34, pp. 501 - 516, 2014. [Online]. Available: http://www.sciencedirect.com/science/article/pii/S1364032114001920

[15] A. Di Giorgio, A. Giuseppi, F. Liberati, and A. Pietrabissa, "Controlled electricity distribution network black start with energy storage system support," in 2017 25th Mediterranean Conference on Control and Automation (MED), July 2017, pp. 781-786.

[16] A. Di Giorgio, A. Giuseppi, F. Liberati, A. Ornatelli, A. Rabezzano, and L. R. Celsi, "On the optimization of energy storage system placement for protecting power transmission grids against dynamic load altering attacks," in 2017 25th Mediterranean Conference on Control and Automation (MED), July 2017, pp. 986-992.

[17] J. Rivera, C. Goebel, and H.-A. Jacobsen, "A distributed anytime algorithm for real-time ev charging congestion control," in Proceedings of the 2015 ACM Sixth International Conference on Future Energy Systems, ser. e-Energy '15. New York, NY, USA: ACM, 2015, pp. 67-76.

[18] S. Studli, E. Crisostomi, R. Middleton, J. Braslavsky, and R. Shorten, Distributed Load Management Using Additive Increase Multiplicative Decrease Based Techniques. Singapore: Springer Singapore, 2015, pp. 173-202. [Online]. Available: https://doi.org/10. 1007/978-981-287-302-6_7

[19] B. Jiang and Y. Fei, "Decentralized scheduling of pev on-street parking and charging for smart grid reactive power compensation," in 2013 IEEE PES Innovative Smart Grid Technologies Conference (ISGT), Feb 2013, pp. 1-6. 
[20] O. Ardakanian, C. Rosenberg, and S. Keshav, "Distributed control of electric vehicle charging," in Proceedings of the Fourth International Conference on Future Energy Systems, ser. e-Energy '13. New York, NY, USA: ACM, 2013, pp. 101-112. [Online]. Available: http://doi.acm.org/10.1145/2487166.2487178

[21] W. Qi, Z. Xu, Z. M. Shen, Z. Hu, and Y. Song, "Hierarchical coordinated control of plug-in electric vehicles charging in multifamily dwellings," IEEE Transactions on Smart Grid, vol. 5, no. 3, pp. 14651474, May 2014.

[22] X. Luo, S. Xia, and K. W. Chan, "A decentralized charging control strategy for plug-in electric vehicles to mitigate wind farm intermittency and enhance frequency regulation," Journal of Power Sources, vol. 248, pp. $604-614,2014$.

[23] A. Malhotra, G. Binetti, A. Davoudi, and I. D. Schizas, "Distributed power profile tracking for heterogeneous charging of electric vehicles," IEEE Transactions on Smart Grid, vol. 8, no. 5, pp. 2090-2099, Sep. 2017.

[24] P. Kong and G. K. Karagiannidis, "Charging schemes for plug-in hybrid electric vehicles in smart grid: A survey," IEEE Access, vol. 4, pp. 6846-6875, 2016.

[25] M. Amjad, A. Ahmad, M. H. Rehmani, and T. Umer, "A review of evs charging: From the perspective of energy optimization, optimization approaches, and charging techniques," Transportation Research Part D: Transport and Environment, vol. 62, pp. 386 - 417, 2018. [Online]. Available: http://www.sciencedirect.com/science/article/pii/ S1361920917306120

[26] Y. Shi, H. D. Tuan, A. V. Savkin, T. Q. Duong, and H. V. Poor, "Model predictive control for smart grids with multiple electric-vehicle charging stations," IEEE Transactions on Smart Grid, vol. 10, no. 2, pp. 2127-2136, March 2019.

[27] G. Wenzel, M. Negrete-Pincetic, D. E. Olivares, J. MacDonald, and D. S. Callaway, "Real-time charging strategies for an electric vehicle aggregator to provide ancillary services," IEEE Transactions on Smart Grid, vol. 9, no. 5, pp. 5141-5151, Sep. 2018.

[28] A. D. Giorgio, F. Liberati, and S. Canale, "Electric vehicles charging control in a smart grid: A model predictive control approach," Control Engineering Practice, vol. 22, pp. 147 - 162, 2014. [Online]. Available: http://www.sciencedirect.com/science/article/pii/S0967066113001871

[29] A. S. Gazafroudi, J. M. Corchado, A. Keane, and A. Soroudi, "Decentralised flexibility management for evs," IET Renewable Power Generation, vol. 13, pp. 952-960(8), April 2019. [Online]. Available: https://digital-library.theiet.org/content/journals/10.1049/ iet-rpg.2018.6023

[30] E. Xydas, C. Marmaras, and L. M. Cipcigan, "A multi-agent based scheduling algorithm for adaptive electric vehicles charging," Applied Energy, vol. 177, pp. 354 - 365, 2016. [Online]. Available: http://www.sciencedirect.com/science/article/pii/S0306261916306286

[31] M. A. Tajeddini and H. Kebriaei, "A mean-field game method for decentralized charging coordination of a large population of plug-in electric vehicles," IEEE Systems Journal, vol. 13, no. 1, pp. 854-863, March 2019.

[32] D. Mayne, J. Rawlings, C. Rao, and P. Scokaert, "Constrained model predictive control: Stability and optimality," Automatica, vol. 36, no. 6, pp. 789 - 814, 2000. [Online]. Available: http://www.sciencedirect.com/science/article/pii/S0005109899002149

[33] C. Bruni, F. Delli Priscoli, G. Koch, A. Palo, and A. Pietrabissa, "Quality of experience provision in the future internet," IEEE Systems Journal, vol. 10, no. 1, pp. 302-312, March 2016.

[34] A. Pietrabissa, F. Delli Priscoli, A. D. Giorgio, A. Giuseppi, M. Panfili, and V. Suraci, "An approximate dynamic programming approach to resource management in multi-cloud scenarios," International Journal of Control, vol. 90, no. 3, pp. 492-503, 2017. [Online]. Available: https://doi.org/10.1080/00207179.2016.1185802

[35] J. Borwein and A. S. Lewis, Convex analysis and nonlinear optimization: theory and examples. Springer Science \& Business Media, 2010. 